\title{
An Energy Efficient Interference-aware Routing Protocol for Underwater WSNs
}

\author{
Anwar Khan ${ }^{1}{ }^{* *}$, Nadeem Javaid ${ }^{2}$, Ihsan $\mathrm{Ali}^{3}$, Mohammad Hossein Anisi ${ }^{4}$, Atiq Ur Rahman ${ }^{5}$, \\ Naeem Bhatti ${ }^{6}$, Muhammad Zia ${ }^{7}$ and Hasan Mahmood ${ }^{8}$ \\ ${ }^{1}$ Department of Electronics, University of Peshawar \\ Peshawar, KPK 25000 - PAK \\ [e-mail: arkhan@upesh.edu.pk] \\ ${ }^{2}$ Department of Computer Science, COMSATS Institute of Information Technology \\ Islamabad, 44000 - PAK \\ [e-mail: nadeemjavaidqau@gmail.com] \\ ${ }^{3}$ Department of Computer System and Technology, Faculty of Computer Science and Information Technology \\ University of Malaya \\ Kualalumpur, 50603 - MY \\ [e-mail: ihsanalichd@gmail.com] \\ ${ }^{4}$ School of Computer Science and Electronic Engineering, University of Essex, \\ Colchester, CO4 3SQ, United Kingdom. \\ [e-mail: anisi@gmail.com, anisi@ieee.org] \\ ${ }^{5}$ Faculty of Computing and Information Technology, Northern Border University \\ Rafha, 11351 - KSA \\ [e-mail: atiq621@gmail.com] \\ 6, 7, 8, ** Department of Electronics, Quaid-i-Azam University \\ Islamabad, 44000 - PAK \\ [e-mail: nbhatti@qau.edu.pk] ${ }^{6}$, [e-mail: mzia@qau.edu.pk] , [e-mail: hasan@qau.edu.pk] ${ }^{8}$ \\ *Corresponding author: Anwar Khan
}

Received Jaunuary 31, 2017; revised May 3, 2017; accepted June 17, 2017;

published October 31, 2017

\begin{abstract}
Interference-aware routing protocol design for underwater wireless sensor networks (UWSNs) is one of the key strategies in reducing packet loss in the highly hostile underwater environment. The reduced interference causes efficient utilization of the limited battery power of the sensor nodes that, in consequence, prolongs the entire network lifetime. In this paper, we propose an energy-efficient interference-aware routing (EEIAR) protocol for UWSNs. A sender node selects the best relay node in its neighborhood with the lowest depth and the least number of neighbors. Combination of the two routing metrics ensures that data packets are forwarded along the least interference paths to reach the final destination. The proposed work is unique in that it does not require the full dimensional localization information of sensor nodes and the network total depth is segmented to identify source, relay and neighbor nodes. Simulation results reveal better performance of the scheme than the counterparts DBR and EEDBR techniques in terms of energy efficiency, packet delivery ratio and end-to-end delay.
\end{abstract}

Keywords: interference-aware, energy efficiency, EEIAR, underwater WSNs, routing 


\section{Introduction}

The design of interference-aware routing protocols for underwater wireless sensor networks (UWSNs) has been one of the subjects of research in recent years. They provide optimal data traffic in accordance with the design parameters from bottom of ocean to the surface of water. Compared to their terrestrial counterparts [1][2], there are a number of challenges inherently associated with all underwater routing protocols; long multi-path delay, low bandwidth and limited battery power [3][4][5]. Underwater routing protocols are used in a number of applications. Specifically, they are used in offshore exploration [6], leak detection, seismic and equipment monitoring [7], military surveillance, underwater navigation, disaster prevention and environmental monitoring [8].

Interference-aware routing protocols are particularly important because of two reasons. Firstly, these protocols improve the quality of underwater communication that is affected by interference. The received data packets at destination do not require rigorous treatment for extracting the desired information. This, in turn, reduces the complexity in the final destination circuitry. It is because extraction of the desired information from the received data becomes easier and, therefore, does not require sophisticated devices. In other words, it shortens the processing time of devices in interpretation of the information after the received data is input to them. This shortening of the processing time is of significant importance in delay sensitive applications. Secondly, they avoid paths with excessive interference in routing packets from source to destination. This reduces packets drop and collisions, which consequently, increases the probability of successful packet delivery at destination. Packets drop due to interference is one of the serious threats to the limited battery power of nodes in underwater communication. It also leads to loss of information and critical data that are always undesired, especially in data sensitive applications. Long and persistent operation of nodes demands that their energy is utilized in an efficient fashion.

The conventional depth-based routing (DBR) protocol [9] and other routing techniques such as directional flooding based routing (DFR) [10], vector-based forwarding (VBF) [11], hop-by-hop vector-based forwarding (HH-VBF) [12] and focused beam routing (FBR) [13] route the packets from source to destination using flooding. However, it leads to redundant packets transmission, interference and packets collision. This, in turn, causes unnecessary energy consumption to a significant extent. Also, with depth as the routing metric, the low depth nodes are overburdened due to frequent selection as forwarders which soon leads to energy holes formation. Creation of holes results in packets loss as it reduces the probability of finding a forwarder node. Energy efficient depth-based routing (EEDBR) protocol [14]; although reduces energy consumption in DBR, it also suffers from the early death of high energy nodes that are close to the sink. Death of such nodes increases packets loss. Although DBR makes use of packet history buffer and packet holding time to avoid redundant packets transmission and interference, forwarding packets in the flooding manner does not appreciably overcome them as every node receives packets from all nodes within its transmission range or within the depth threshold. It then transmits the packet further in a greedy manner. It is because when a source node sends a packet, all its alive one-hop neighbors receive it. This packet is further forwarded towards the sink by neighboring nodes that are at lower depth than the original source node (greedy). In other words, a single packet is unnecessarily forwarder by more than one forwarder. As a result, redundant packets transmission increases that finally results in interference.

Design of interference-aware routing protocols carries a number of challenges. The 
underwater medium is highly time varying and fluctuating [15], therefore the packets transmitted towards destination may be badly affected by its properties. This may include reflection, refraction and diffraction of the signal from water molecules, underwater objects, noise and shadow zones therein. Also, redundant packets transmission to successfully send packets from source to destination; even if some packets drop along the routing path, causes additional interference and packets collision. If such redundant packets are not properly coped with, this results in packet loss. Further, sensor nodes change their positions with water currents making localization of nodes a cumbersome task. According to the DBR protocol [9], localization is the full-dimensional information of a sensor node. That is, localization requires that the $x, y$ and $z$ coordinates of a sensor node are known. A sender node can only calculate the distance between itself and the best forwarder if it knows all the three coordinates of itself and of the forwarder. However, it consumes extra amount of energy by implementing a localization technique that periodically measures all the coordinates of nodes. The proposed work addresses some of these challenges.

In this paper, we propose EEIAR; an energy efficient and interference-aware routing protocol for UWSNs that avoids interference in routing packets from a sender node to a receiver node. The protocol selects forwarder nodes that have the least number of neighbors and the lowest depth. Choosing such a forwarder node avoids interference in transmission and reception of packets. The lowest depth ensures that packets come closer to the surface sink after each transmission. Unlike forwarding the packets in a flooding fashion, a sender node decides and selects the best forwarder node. Such a decision reduces energy consumption, packets scollision and packets drop by controlling redundant packets transmission. The choice of selection of a forwarder node by a sender node further allows it to select forwarder nodes within its full transmission range. This is contrary to most of the flooding based routing protocols that usually select forwarder nodes within fixed regions to reduce energy consumption and redundant packets transmission. As a result, the probability of unavailability of a forwarder node reduces. This, as a consequence, increases the probability of successful packets received at the sink. Also, the EEIAR does not require the full-dimensional localization information of nodes. It requires only the depth information of a sender and a forwarder to route data to the final destination. The depth of a node is its vertically downward distance from the surface of water and does not require the other two coordinates of the node to be known. In other words, it does not require the full-dimensional information of a node. In contrary, the depth information can be obtained by a pressure sensor attached with a sensor node. Greater vertical distance in the downward direction from the surface of water means more water pressure that, in turn, means greater depth. It does not require any localization technique separately to identify complete coordinates of nodes.

\section{Related Work}

In this section, a description of interference-aware routing protocols is given for UWSNs. The authors in [16] propose interference-aware routing and scheduling policies for sensor nodes to achieve energy efficiency in order to efficiently utilize the available bandwidth in underwater communication. The scheduling policies give priority to nodes that are capable for earlier transmission of packets than others, have greater number of packets in their buffers, are positioned farther from the sink and have sent less number of packets. These policies result in minimized time difference between the readiness of a packet for transmission and its effective transmission time. They also result in reduction of the path from source to destination, buffer size of forwarder nodes and data traffic. Further, packets are transmitted with varied set of 
power levels. Various combinations of these scheduling and routing policies are combined to obtain the optimal results. However, the proposed system is based on too many assumptions in the routing and scheduling policies that make it less practical to implement. The work in [17] designs a routing protocol that avoids interference and hole formation for reliable data transfer. A sender or source node that has packets to send selects a potential forwarder node among its neighbors. It first calculates a cost function based on the number of neighbors of the potential forwarder and its distance from the sender and hop count from the sink. Packets are then forwarded to the potential forwarder node that has the highest cost function. However, the calculation of distance involves localization of sensor nodes that limits its applicability. Localization of sensor nodes is generally difficult to implement and expensive in underwater communication as nodes have to constantly update and share their location information. Mahreen et al [18] propose three protocols: inverse EEDBR (IEEDBR), interference-aware EEDBR (IA-EEDBR) and interference-aware inverse energy efficient EEDBR (IA-IEEDBR). These protocols improve the delay, energy consumption, network stability period, path loss and transmission loss of EEDBR. In IEEDBR, a sender node selects a forwarder node within its transmission range with the lowest depth and the least amount of residual energy. However, such nodes die early that creates holes in the network and badly affects system performance. In IA-EEDBR, a forwarder node is selected having the highest residual energy, lowest depth and the least number of its neighbors. However, just like EEDBR, high energy nodes die as the routing process continues. In IA-IEEDBR, a forwarder node having the least residual energy, lowest depth in the specified depth threshold rather than in the full transmission range and the least number of neighbors forwards the packet. Again, holes are created when low energy nodes die.

The authors in [19] propose an improved interference-aware EEDBR (iIA-EEDBR) protocol to avoid creation of holes and prolong network lifetime and the number of packets received at the sink. Half of the nodes are deployed in sensing mode and the rest in sleeping mode. The network is segmented into four logical sections based on depth. Every section has a header node with which the sleeping nodes exchange their depth, ID and section number. When a sensing node in a section dies, the header node turns a sleeping node into a sensing node. The protocol works in two stages. In the first stage, nodes exchange information about their depth and residual energy with their neighbors. During the second data transmission stage, sender nodes forward data packets to neighbor nodes that have the highest residual energy, the least number of neighbors and the lowest depth. However, the performance of this protocol severely degrades when the header node dies due to constant monitoring of sensing and sleeping nodes and taking part in the routing process. In addition, a node may die in one location and a sleeping node may become active in a different location. So, it may not actively counteract the effect of the death of a sensing node. The work in [20] proposes energy balanced interference-aware EEDBR (EB-IAEEDBR) that balances energy consumption in IEEEDBR protocol. Initially, all nodes are assigned equal amount of energy level called energy grade. As the protocol operates, the energy grade of nodes varies. When a sender node has to send packets, it checks for the energy grade of the next expected forwarder. If the energy grade of the sender is less than or equal to that of the receiving node, it forwards the packet to the expected receiving node that further forwards it in the same manner. When the energy grade of a node falls below a threshold, it transmits a control packet to one-hop neighbors. The neighbors receive it and start direct transmission towards the sink. So, the protocol achieves energy balancing by not choosing forwarder nodes that have energy below a certain threshold. The protocol switches from the initial multi-hop communications to direct transmission when high energy nodes start to die (as is the case with EEDBR). An energy efficient and 
interference-aware routing protocol is proposed in [21]. The three-dimensional (3D) network is segmented into three zones: top destination region, mid relay region and the bottom source region. A source node in the bottom region selects a relay node in the mid region with the least distance from the sink and the least number of neighbors. The protocol improves energy consumption, packets reception at the sink and end-to-end delay. However, it requires localization information of sensor nodes. The authors in [22] propose a channel-aware routing protocol that considers the speed of sound and the channel noise with respect to depth to route packets from source to destination. The protocol functions in two modes. The collecting mode (CM) in which nodes share neighbors information and the direct mode (DM) in which a sender node forwards data to a forwarder node. A source node first constructs an ideal virtual path to the sink and then calculates a weighting function for every forwarder node based on the probability of successful transmission, the distance between candidate nodes and destination and the distance between the candidate nodes and the ideal path. Forwarder with the highest weighting function is then selected for data forwarding. The protocol outperforms the counterpart scheme in terms of packet delivery ratio and end-to-end delay. However, the protocol involves distance calculation that constraints its application.

The authors in [23] propose two protocols: energy hole repairing DBR (EHRDBR) and interference-bandwidth-aware DBR (IBDBR). The former selects forwarder nodes based on the interference, residual energy and depth while the latter chooses them by considering interference, bandwidth, residual energy and depth. In both protocols, when a node dies, a live node moves to its location to avoid hole creation. These protocols show better performance than the counterpart schemes in terms of end-to-end delay, packets received at the sink and network lifetime. However, locating the position of a dead node and replacing it with a live one requires location information and is troublesome to do, especially when nodes are not stationary and move with the water currents. In addition, considering too many parameters for forwarder selection complicates the internal structure of the sensor nodes.

\section{Channel Model}

The process of data routing in aquatic environment is severely affected by the properties of underwater channel.

\subsection{Channel Noise}

Underwater noise adds up to the desired signal and badly affects its quality. In underwater communications, the ambient noise consists of four types: wave, shipping, turbulence and thermal noise [24]. Its power spectral density (PSD) $N$ in $\mathrm{dB}$ is given by

$$
N=N_{t}+N_{s}+N_{w}+N_{t h}
$$

where $N_{t}, N_{s}, N_{w}$ and $N_{t h}$ express the PSDs of turbulence, shipping, wave and thermal noise, respectively. Their power spectral densities are characterized by [24]

$$
\begin{gathered}
N_{s}=40+20(s-0.5)+26 \log f-60 \log (f+0.03) \\
N_{w}=50+7.5 w^{0.5}+20 \log f-40 \log (f+0.4) \\
N_{t}=27-30 \log f \\
N_{t h}=-25+\log f
\end{gathered}
$$


where $f$ is the frequency in $\mathrm{kHz}$ and $w$ is the speed of wind in $\mathrm{m} / \mathrm{s}, \mathrm{s}$ is the shipping activity factor. Shipping activities cause shipping noise that dominates in the $20 \mathrm{~Hz}$ to $200 \mathrm{~Hz}$ range. Wind generates waves at the surface of ocean that result in wave noise in the $200 \mathrm{~Hz}-200 \mathrm{KHz}$ range. Turbulence noise affects frequencies smaller than $20 \mathrm{~Hz}$ while thermal noise prevails above $200 \mathrm{kHz}$.

\subsection{Channel Losses}

Attenuation in underwater communication is the result of channel losses that consume the battery power of nodes [25]. The most prominent are absorption and transmission losses. Absorption Loss: When an acoustic wave travels in water, it dissipates energy as heat due to friction and ionic relaxation and constitutes absorption loss given by

$$
\begin{gathered}
P L_{\text {absorption }}=\left(\alpha \times 10^{-3}\right) r \\
P L_{\text {spreading }}=k \log r
\end{gathered}
$$

where $P L_{\text {spreading }}$ is the path loss in $\mathrm{dB}$ due to spreading, $r$ is the transmission range in meter and $k$ is the spreading factor and represents the geometry of the spread ( $k=1$ for cylindrical spreading and $k=2$ for spherical spreading).

\subsection{Speed of Acoustic Wave}

The radio frequency waves are attenuated to a significant extent in water. Therefore, they are not used in underwater communications. Instead, acoustic waves are used. The speed $c$ of these waves depends upon the channel properties and is modeled [26] as

$$
\begin{aligned}
& c= 1449+4.591 T-5.304 \times 10^{-2} T^{2}+2.374 \times 10^{-4} T^{3}+1.34(S-35)+1.63 \\
& \times 10^{-2} D+1.675 \times 10^{-7} D^{2}+1.025 \times 10^{-2} T(S-35)-7.139 \\
& \times 10^{-3} T D^{3}
\end{aligned}
$$

where $T$ is the temperature in degree Celcius, $D$ is the depth of water in meter and $S$ is the salinity factor in parts per thousand.

\subsection{Bandwidth and Convergence Zone}

In underwater communications, the available bandwidth is kept limited by channel properties such as noise, losses and long multi-path delay. It varies significantly with the distance from source to destination as shown in the Table 1 [8]. It is clear that as the covergence (also called transmission range) decreases, bandwidth increases. Since bandwidth is the maximum data that can be transmitted along the channel in a given time, a decrease in the bandwidth generally decreases the data rate as well. According to the nature of application, different applications use different range and bandwidth. In general, a depth of $100 \mathrm{~m}$ or smaller is considered as shallow and uses the maximum available bandwidth. Applications such as ocean bed monitoring, rock and debris detection use more depth so the available bandwidth is smaller for them. 
Table 1. Bandwidth and convergence relationship in UWSNs.

\begin{tabular}{|c|c|c|}
\hline Convergence & Range $[\mathrm{km}]$ & Bandwidth $[\mathrm{kHz}]$ \\
\hline Very long & 1000 & Less than 1 \\
\hline Long & $10-100$ & $2-5$ \\
\hline Medium & $1-10$ & Almost 10 \\
\hline Short & $0.1-1$ & $20-50$ \\
\hline Very short & Less than 0.1 & Greater than 100 \\
\hline
\end{tabular}

\section{The Proposed Protocol}

\subsection{Network Architecture}

The proposed network is a three dimensional cube with $500 \mathrm{~m}$ length of a face. Deployment of sensor nodes is accomplished in a random fashion with the sink localized at the top middle of the network. In order to distinguish among source, relay and destination nodes, the network total depth $D$ is generally segmented into three equal sectors or regions as shown in Fig. 1.

The topmost region is called destination region as it lies close to the sink (destination). Nodes in this sector are called destination nodes. The mid sector is called relay or forwarder region. We use the terms relay and forwarder interchangeably unless stated otherwise. Nodes in the bottom source sector are called source nodes. Source nodes in the bottom region forward data to relay nodes that further forward it to the destination nodes. From the destination region, data is sent to the surface sink. The bottom nodes are called source nodes because they generally sense the desired attribute. The routing among nodes in these regions is described in more detail in the data forwarding phase in section 4.3. All nodes communicate with one another through acoustic links as radio waves are attenuated to a greater extent in underwater communications. The sink, on the other hand, communicates with the onshore data center through a radio link and with sensor nodes through the acoustic links.

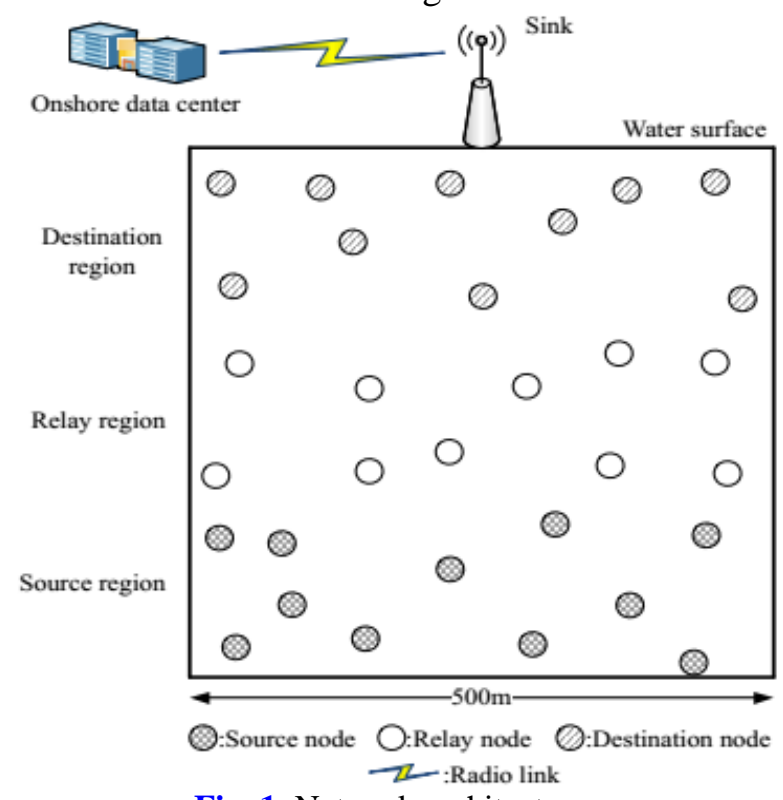

Fig. 1. Network architecture. 
Due to the greater speed of radio waves than the acoustic waves, we assume that data packets that reach to the sink are considered successfully delivered to the onshore data center.

\subsection{Neighbors Identification}

After deployment of nodes, initially they do not know about the depth and neighbors information of one another. Every node broadcasts a hello message. All other nodes that lie within its transmission range receive it. The message contains the depth information and ID of the broadcasting node. Every node within the range replies to the hello message of the broadcasting node that processes the received messages and gains information about its neighbors. Every node then constructs a table of its neighbors and broadcasts it. In this fashion, every node is aware of its own neighbors and 2-hop neighbors along with their depth information. The knowledge of depth and number of neighbors helps a sender node to select the best forwarder. The broadcasting node waits for a reply from every neighbor node in response to the hello message for a certain time proportional to the propagation and processing delay in underwater communication. If it does not receive any reply from any node, it sends the hello message again. It declares no neighbor at all when the hello message is sent for the maximum number of times and gets no response within the specified waiting time. All nodes periodically exchange the hello messages and the neighbor tables to remain updated about the alive number of neighbors as nodes die due to consumption of their limited battery power as the routing process progresses. In addition, existing neighbor of a node may leave or new may come within its transmission range with water currents.

\subsection{Data Forwarding}

When a node in the source region senses the desired attribute, it creates data packets and chooses the best forwarder node in the mid forwarder region within its transmission range to send the packets to it. The best forwarder has the lowest depth and the least number of neighbors among all the neighbors of the source node. When the source node does not find any forwarder among its neighbors in the forwarder region, it selects a forwarder node among its neighbors in the source region. The selected source node repeats the same strategy as described above to forward packets to the forwarder region. Nodes in the forwarder region further forward the packets to nodes in the destination region that finally forward them to the sink. We assume all nodes are worthy of sensing the desired attribute. At every stage, the best forwarder is selected. Every node sends packet to the best forwarder only when the channel is free. If it is not free, the node backs off. The packet is dropped when the backing off reaches to its maximum limit. Selection of the lowest depth nodes ensures that a data packet becomes closer to the sink after every time it is forwarded by a forwarder node. Choosing a forwarder node with the least number of neighbors reduces the interference and packet collision and, in turn, minimizes packet drop. It contributes to energy efficiency too by reducing the number of nodes receiving the same packet. Decision of forwarding a data packet by a sender node (rather than by a node receiving it) also reduces the number of nodes transmitting the same packet. A node that has to send data chooses another forwarder in its neighborhood based on the same defined criterion when a previous forwarder dies. The death of a sensor node is automatically detected when it does not respond to the periodic hello messages. The ID of a dead node is excluded from the neighboring table that nodes broadcast to identify neighbors. Upon receiving a packet, a forwarder holds it for a particular instant of time termed as the holding time. It depends upon the depth, number of neighbors and the time difference between the reception of two successive packets by the same forwarder. However, every received 
packet is never kept for more than the system characteristic maximum holding time. A timer records the reception time of every packet. If the channel does not become free within the maximum holding time, the packet is declared as dropped. The system characteristic maximum holding time specifies the longest time till a forwarder can hold a received packet. The holding time is made smaller than it (the maximum holding time) so that incoming packets are also received by dropping the already received packets if the channel does not become free until the maximum holding time.

The Fig. 2 shows all the possible cases that a sender node may encounter in forwarding data packets. Of the three nodes A, B and C shown, every node is within the center of a dashed circle that represents its transmission range.

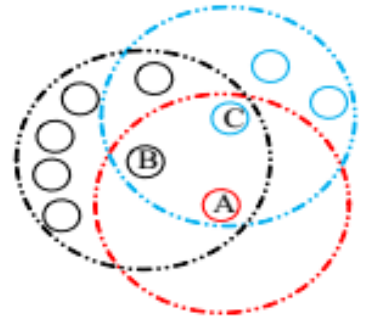

(a)

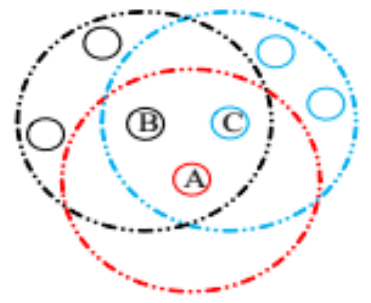

(c)

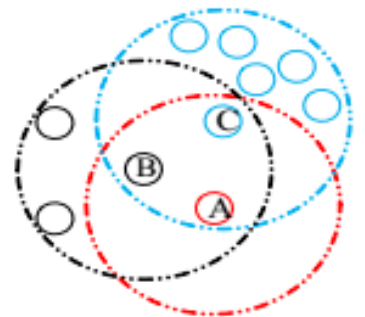

(b)

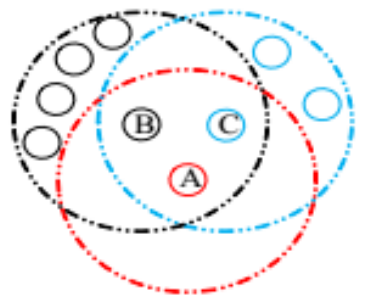

(d)

Fig. 2. Forwarder selection. (a): A chooses C. (b): A chooses B. (c): A chooses either B or C. (d): A chooses C.

In all cases, node $\mathrm{A}$ is the sender of data packets while node $\mathrm{B}$ or $\mathrm{C}$ is the expected forwarder. For simplicity, only two nodes are shown as neighbors of A. In scenario (a), C has lower depth and less number of neighbors than B. Therefore, A chooses C for data forwarding. In case (b), the source node A chooses the greater depth forwarder $B$ than $C$ because of its less number of neighbors. Due to less neighbors, B offers less interference than C, that in turn, reduces the probability of packet drop due to interference although the packet may cover longer path to the sink. In situation depicted in (c), both $B$ and $C$ have the same number of neighbors and depth. Therefore, A may choose either B or C for data routing. Finally, in (d), B and C have the same depth but $\mathrm{C}$ will be chosen to route data due to its less number of neighbors (and less interference).

Algorithm 1 shows selection of the best forwarder node among the neighbors of a sender node. A sender node $i$ selects the best forwarder node from its set of neighbors $N_{i}$. A neighbor node $j$ with the lowest depth and the least number of its neighbors is selected as the best forwarder. This procedure repeats until the packet reaches the sink or is dropped. In Fig. 2(b), source node A chooses the greater depth forwarder B than C because of its less number of neighbors. Due to less neighbors, B offers less interference than C that, in turn, reduces the 
probability of packet drop due to interference although the packet may cover longer path to the sink. The Fig. 3 shows the flow chart that elaborates the operation of the routing protocol. Time $t_{0}$ is the duration by which a forwarder node backs off to the maximum following which the packet is dropped.

A sender node decides the relay node for data forwarding (the sender inserts the ID of the relay node in the data packet to whom it wants to forward it). Therefore, if a packet is received by one or more relays with the same holding time, only the addressed relay will forward it. Others not having their IDs in the data packet will simply discard it. This strategy suppresses the redundant packet transmission yet maintains data delivery.

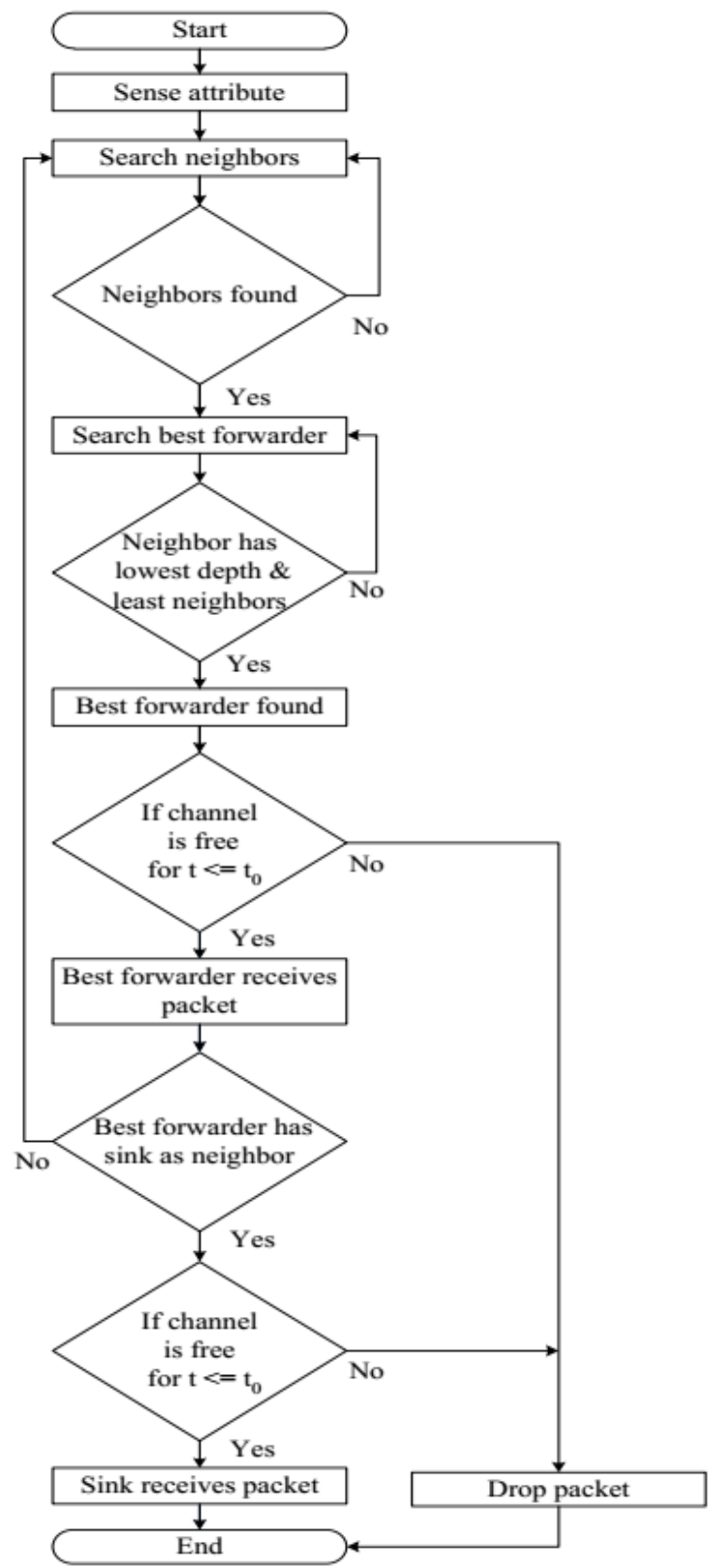

Fig. 3. The flow chart of the routing process. 


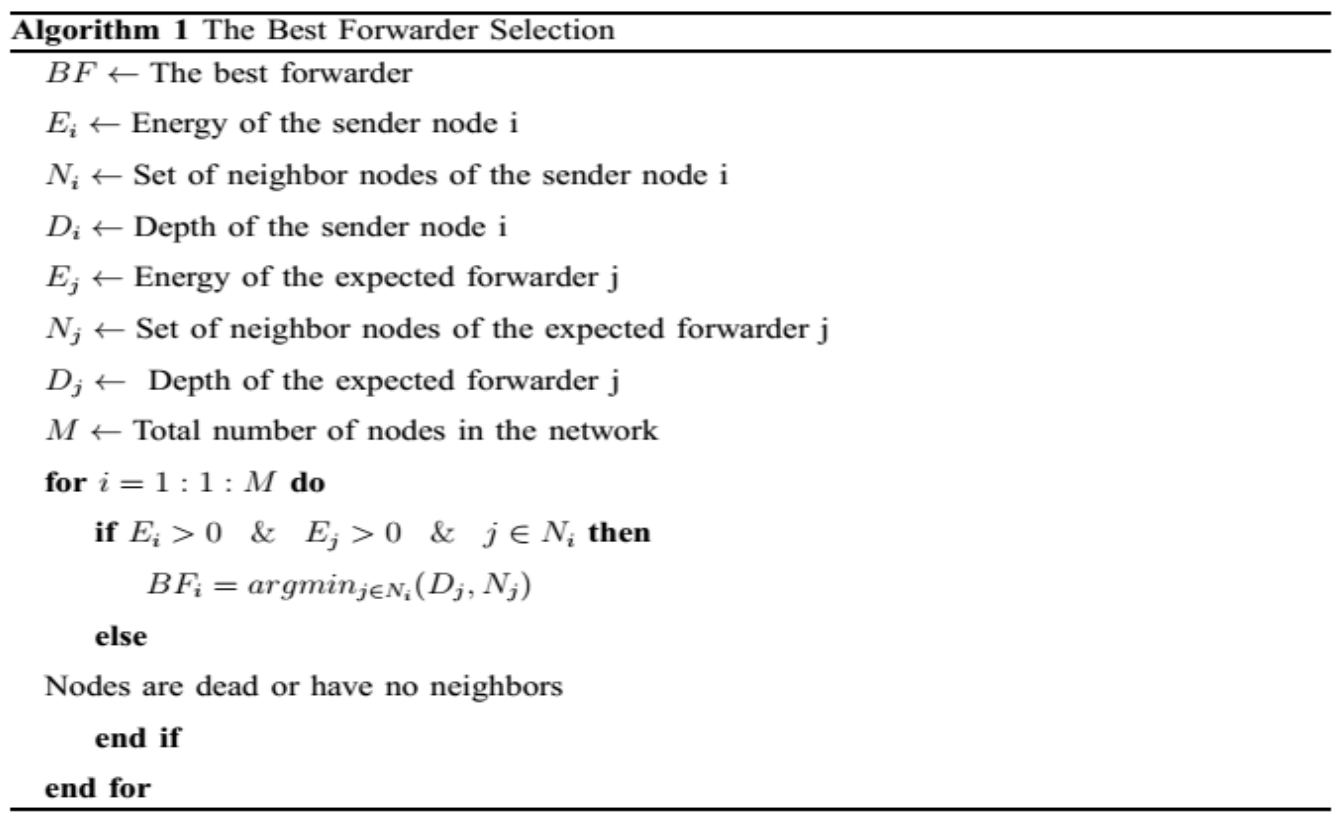

In algorithm 1, the working of min ( $\mathrm{Dj}, \mathrm{Nj}$ ) worths description. A source node looks at the depth values (length) and the number of nodes values (any number greater than or equal to 0 ) of all neighbor nodes. Suppose the source node has three neighbors A, B and C with depth values $200 \mathrm{~m}, 210 \mathrm{~m}$ and $220 \mathrm{~m}$ and with corresponding number of neighbors as 2,4 and 5 , respectively. The first node has the lowest depth of $200 \mathrm{~m}$ and the least number of neighbors (2) so it will be selected as a forwarder by the source node. As mentioned in the neighbor identification phase, every node knows about the depth and number of neighbors of its neighbor nodes. Therefore, it is easy for a sender node to decide a forwarder based on the depth and number of neighbors of the forwarder in its neighborhood. Suppose these three neighbors have the same depth as mentioned above but their number of neighbors become 4, 2 and 3, respectively. Now node A having the lowest depth $(200 \mathrm{~m})$ has the highest number of neighbors so it cannot be selected as the forwarder. Instead, node B with the least number of neighbors will be chosen as the forwarder. This idea is further explained in Fig. $\mathbf{2}$.

\section{Simulation Results and Analysis}

In this section we describe the simulation results and compare the proposed scheme with DBR [9] and EEDBR [14] as they also take into account depth as the routing metric (EEDBR takes into account the residual energy too in deciding forwarder). However, unlike our scheme, they do not take into account the interference mitigation strategy (the least number of neighbors of a forwarder) that leads to packet loss. This work addresses avoidance of interference in a novel way while taking depth as one of the routing parameters. The network is an underwater cube of dimensions $500 \mathrm{~m} \times 500 \mathrm{~m} \times 500 \mathrm{~m}$. We consider random topology, so all the 225 nodes are randomly distributed (deployed) over the three regions of the network. A stationary sink is located at the top middle of the network. We assume that sensor nodes follow the random walk mobility pattern as considered in the DBR protocol. We use the random walk mobility pattern to model the random movement of sensor nodes with water currents. It does not require the 
full-dimensional localization information of sensor nodes as compared to the meandering current mobility model that involves localization [27]. Nodes use the LinkQuest UWM1000 acoustic modem to communicate with one another. The MAC layer is addressed by the 802.11-DYNAV protocol [28]. Every node has a fixed maximum transmission range of $100 \mathrm{~m}$ in all directions and consumes $2 \mathrm{~W}, 0.1 \mathrm{~W}$ and $10 \mathrm{~mW}$ power to transmit, receive and remain in the idle state, respectively. A sensor node generates one data packet per second. The size of a single packet is 50 bytes and the data rate is $10 \mathrm{kbps}$.

Round: The time that lapses from the transmission of a single or more packets by one or more source nodes to its successful reception at the sink or drop.

Total energy consumption: It is the amount of energy consumed by all alive nodes in one round. It may include energy consumption during hello packets exchange, transmission and reception of a packet and while remaining in the idle state.

Dead nodes: Sensor nodes that consume all the initially assigned energy.

Alive nodes: Sensor nodes that have not yet consumed all the initially assigned energy.

End-to-end delay: It is the time taken by a data packet from transmission by source to reception at destination.

Packet delivery ratio: Ratio of total packets received successfully at the sink to total packets transmitted.

Fig. 4 shows plot of the total energy consumption. Due to selection of the path of the least interference (and the lowest depth) for data routing in the proposed scheme, it has the least interference, packet collision and redundant packet transmission as compared to the counterpart schemes. These phenomena contribute to unnecessary consumption of energy that the proposed protocol avoids. In addition, choosing a forwarder node with the least number of neighbors avoids looping a single packet back and forth between neighbors of the forwarder node itself when it transmits the packet further towards destination. It is because with few neighbors, the probability that any two neighbors have the same depth and number of neighbors reduces. If such nodes lie within the transmission range of each other, they may be the forwarder nodes of each other and will send the same packet to one another multiple times. This, in turn, reduces the number of nodes involved in forwarding packets from source to destination. As a result, the EEIAR has the least energy consumption. In contrast, a source node in DBR considers only the lowest depth node as a forwarder. A source node in EEDBR, on the other hand, selects a node as a forwarder that has the lowest depth and the highest residual energy. Despite of packet holding time and history buffer, redundant packet transmission and packet collision are the major issues in the counterpart schemes that are associated with greater energy consumption. EEDBR has lower energy consumption than DBR due to the selection of less forwarder nodes and suppression of the redundant packets transmission than DBR. The Fig. 5 shows plot of the residual energy of sensor nodes. It is the reciprocal of the plot of total energy consumption. The plot of total number of dead nodes is depicted in Fig. 6. On account of the lowest energy consumption, nodes die with the slowest rate in EEIAR as compared to the counterpart schemes. The greater energy consumption in DBR makes its nodes die at a more rapid rate than EEDBR. Fig. 7 shows plot of the number of alive nodes. It is the reciprocal of the plot of dead nodes. 


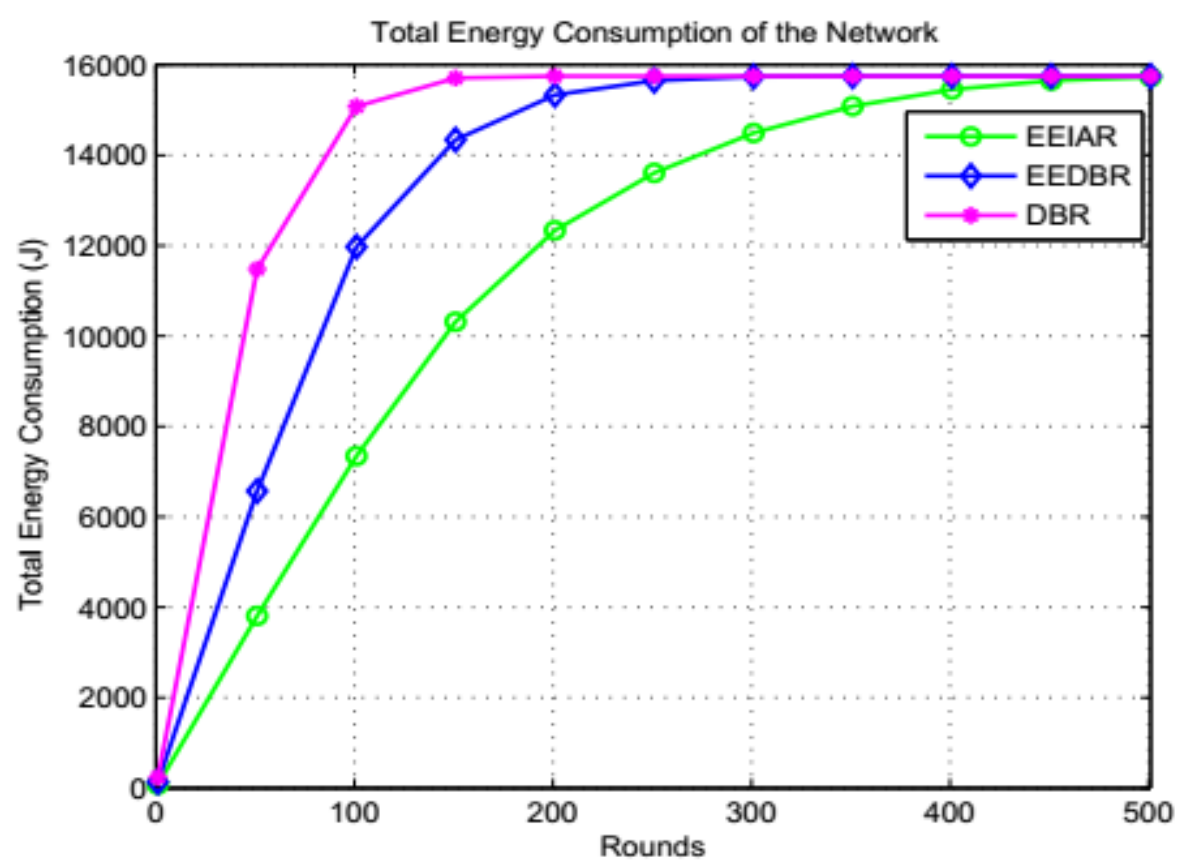

Fig. 4. Total energy consumption in the network.

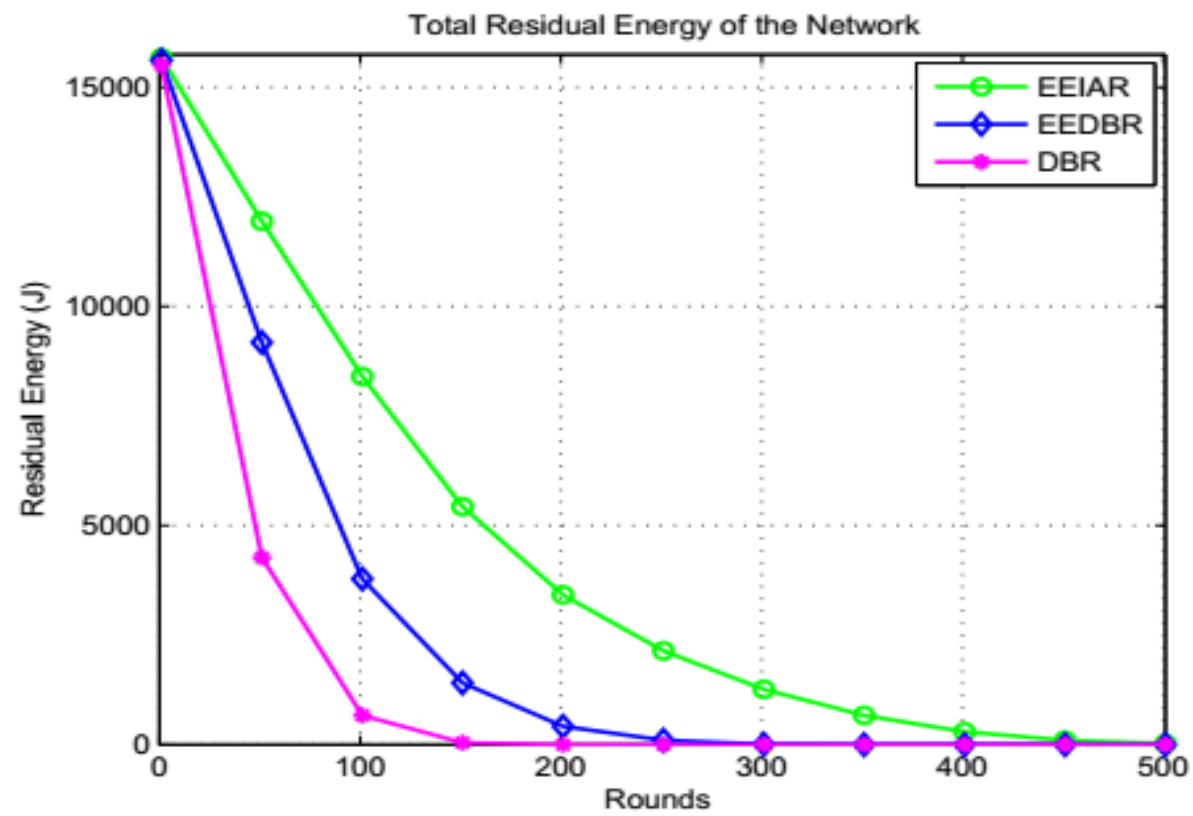

Fig. 5. Total residual energy of the network. 


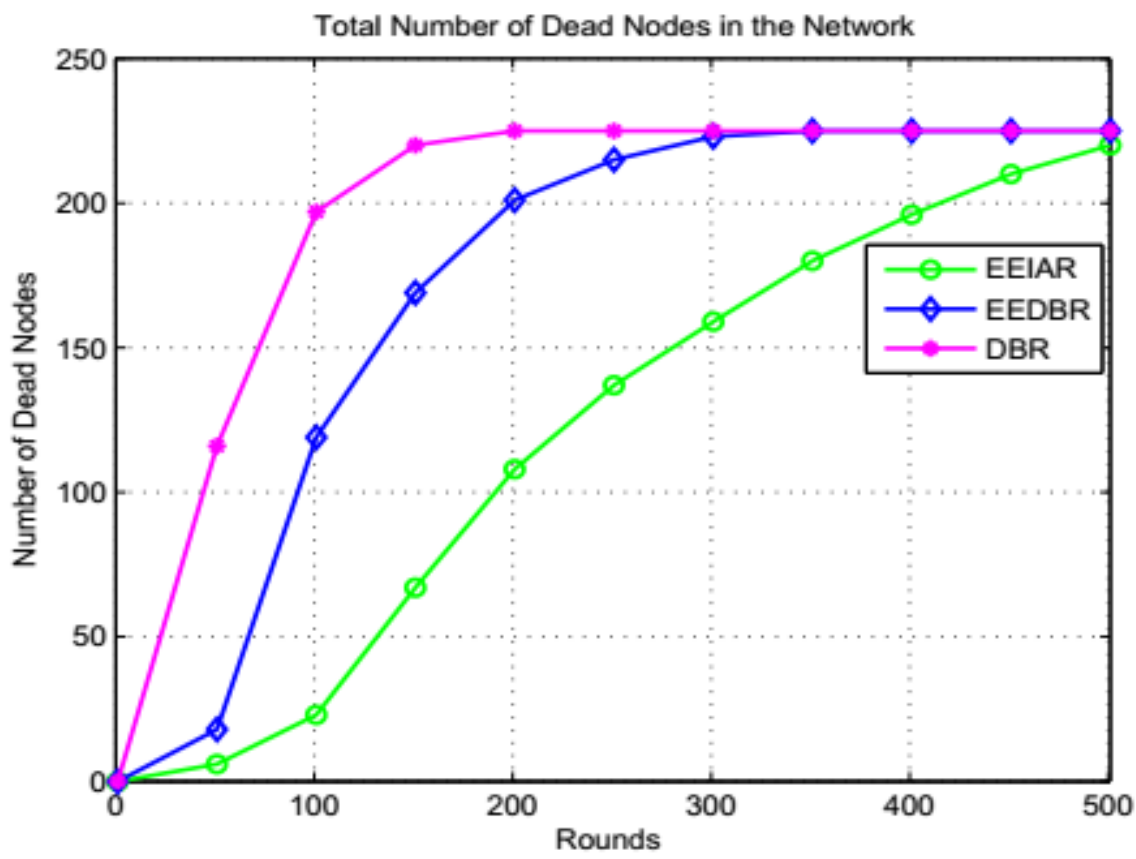

Fig. 6. Total number of dead nodes.

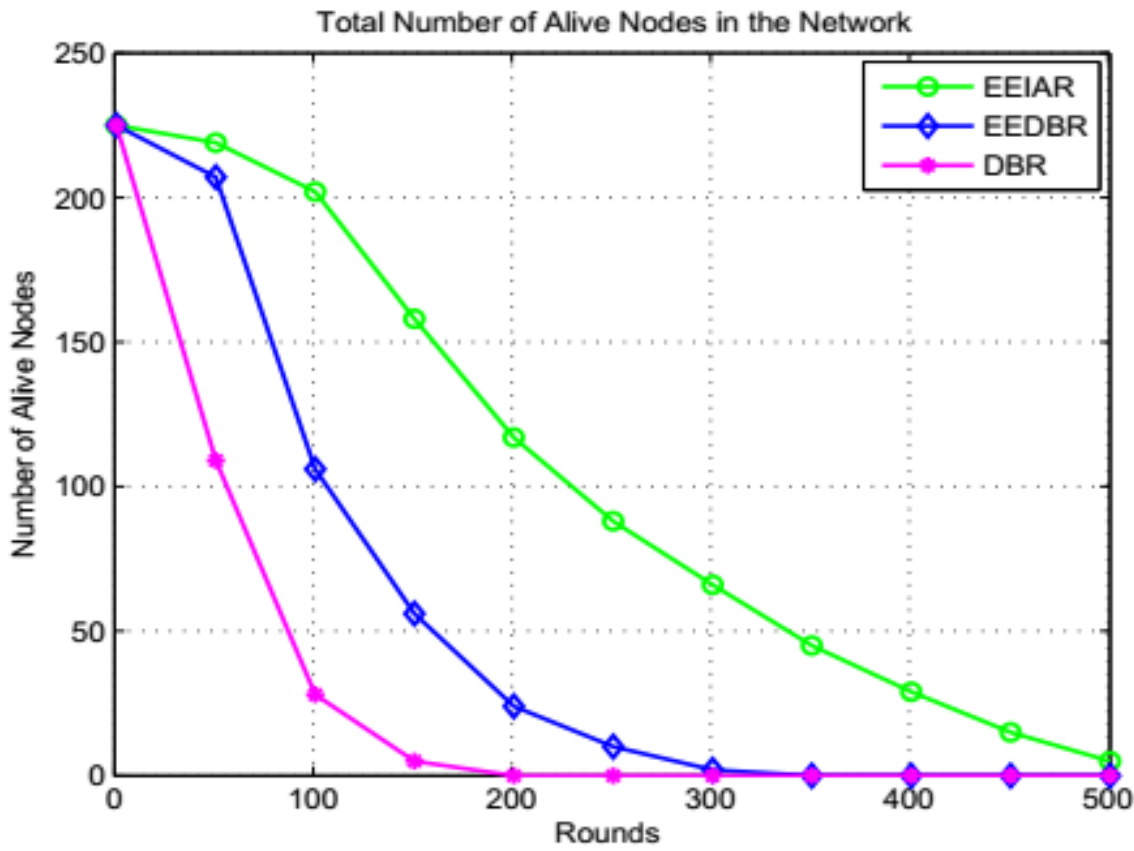

Fig. 7. Total number of alive nodes. 


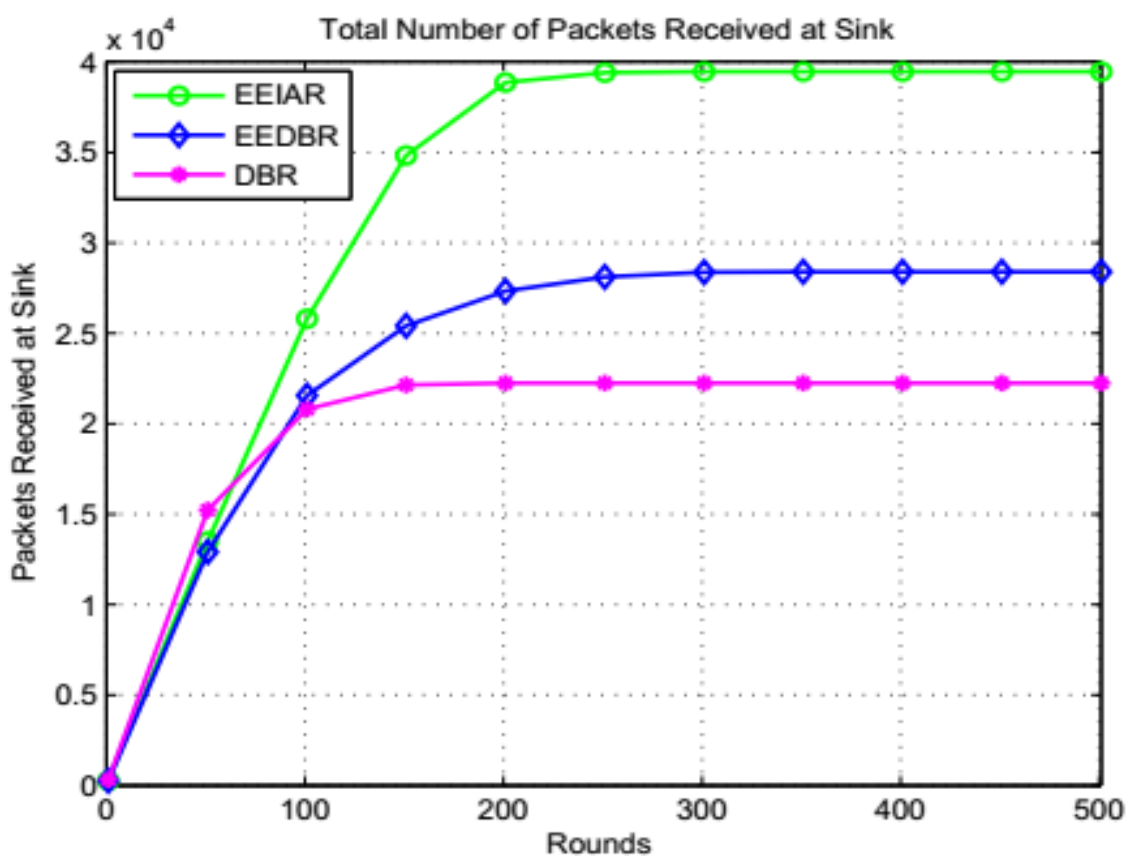

Fig. 8. Total number of packets received at sink.

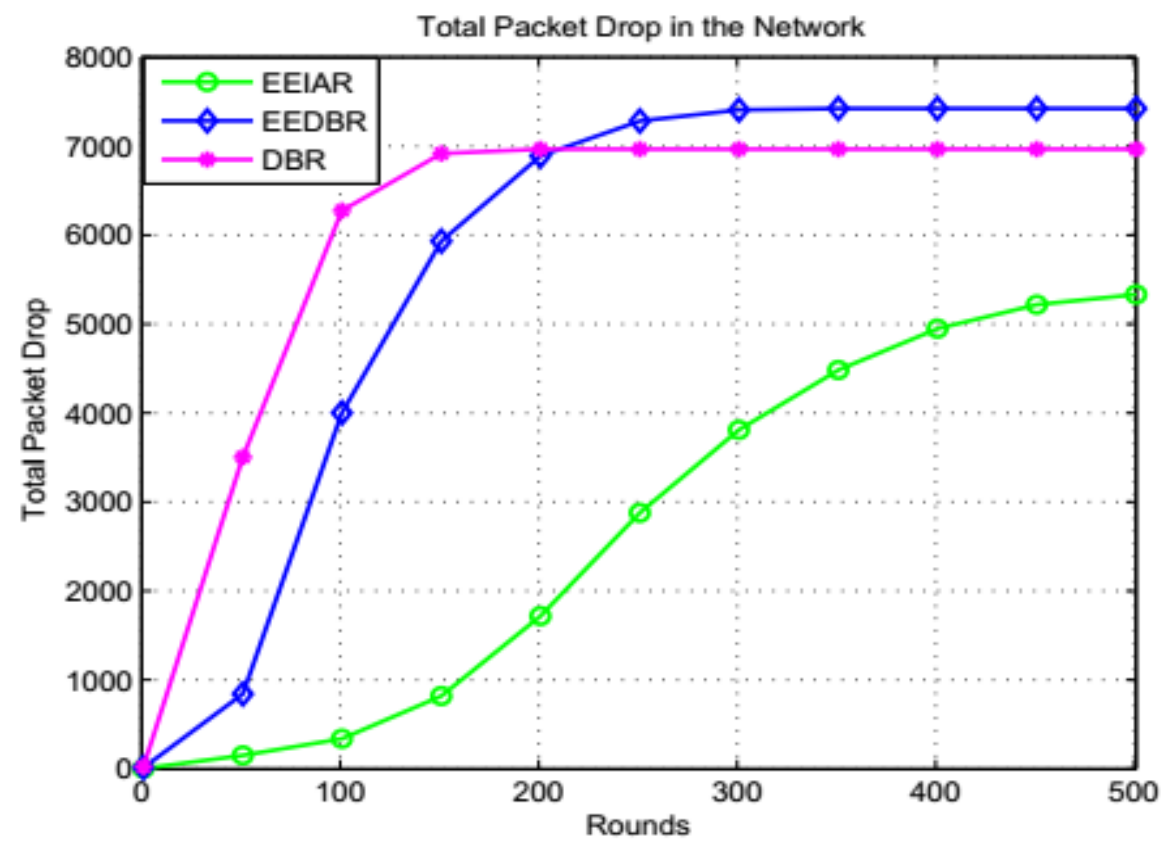

Fig. 9. Total packet drop in the network. 


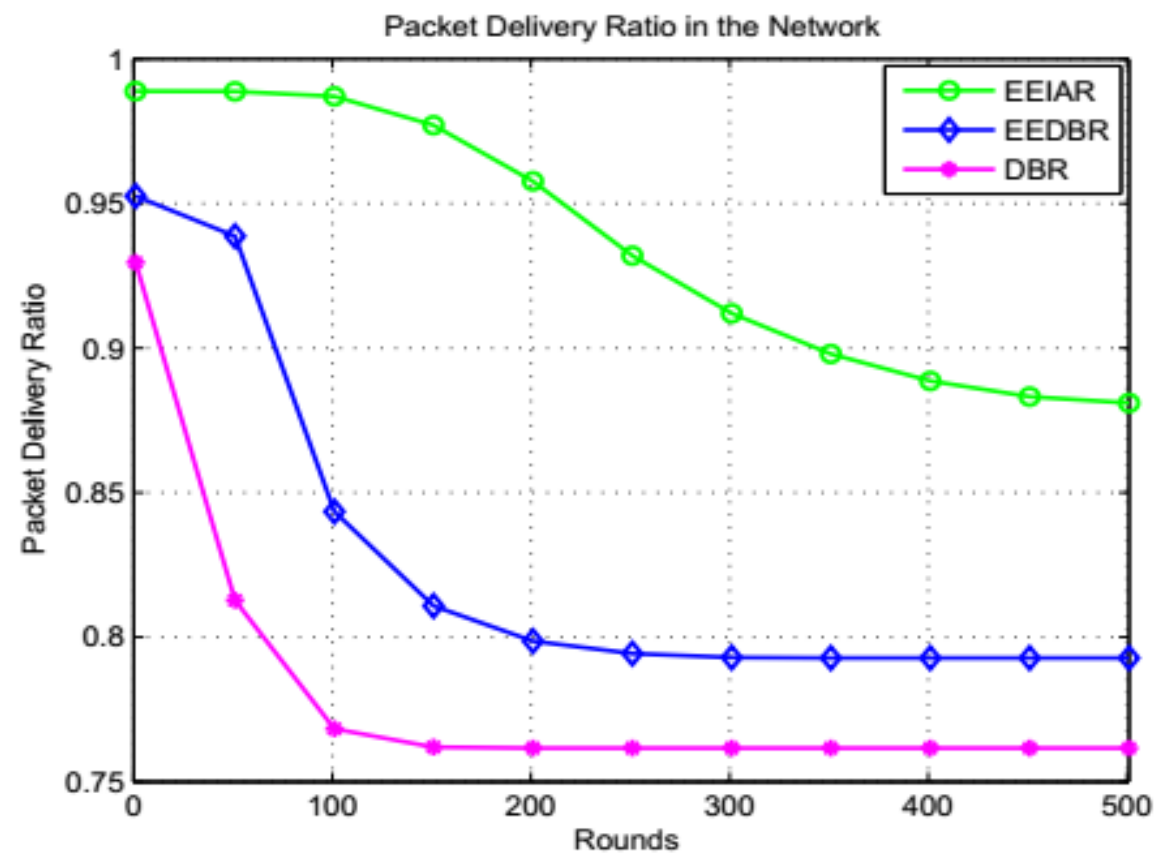

Fig. 10. Packet delivery ratio.

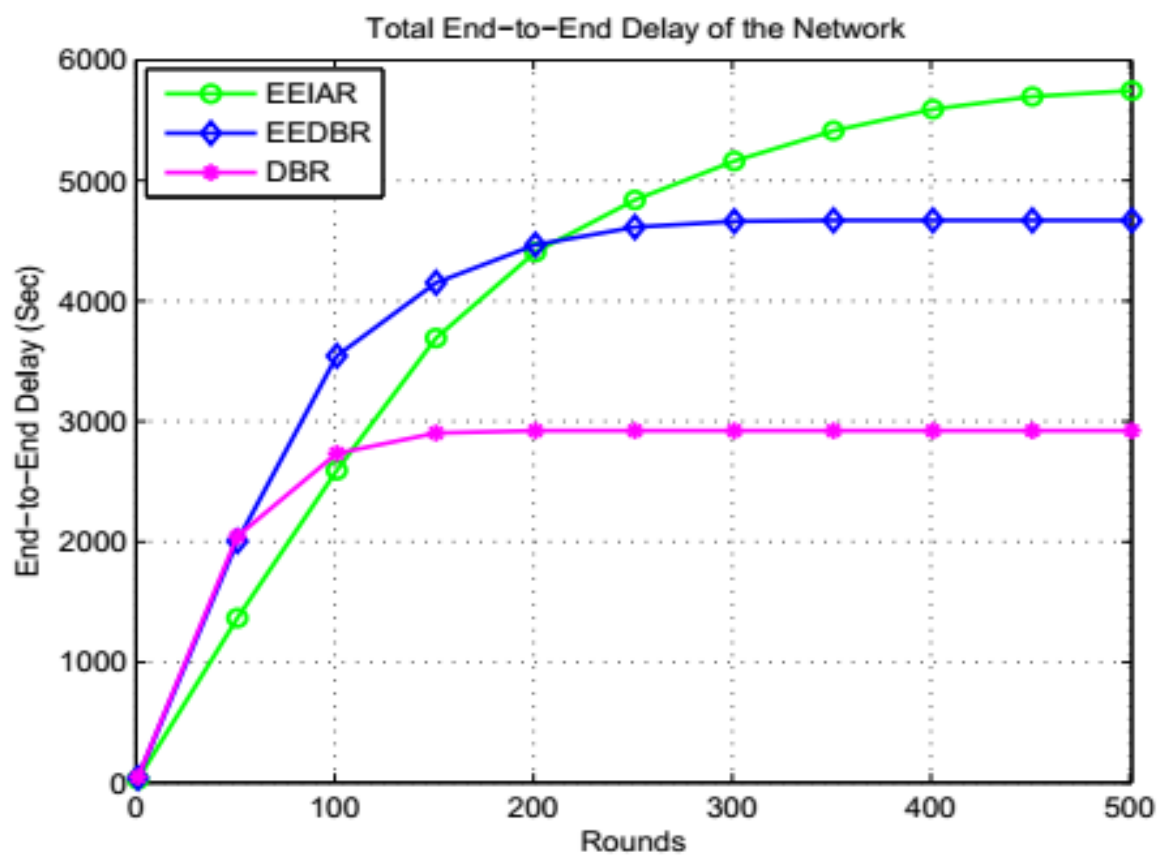

Fig. 11. Total end-to-end delay in the network.

Fig. 8 shows plot of the total number of packets received at the sink. Initially, for almost the first 50 rounds, the number of packets received at the sink is slightly the greatest for DBR. 
It is because DBR chooses the lowest depth nodes that are the closest to the surface of water for packet forwarding. As rounds progress, these nodes overburden and die rapidly. Death of such nodes reduces the availability of forwarder nodes to receive and forward packets to the sink. Consequently, its throughput decreases and becomes the lowest after 100 rounds. The slowest death of nodes and selection of the path of the least interference for data routing in EEIAR ensure the availability of forwarder nodes and its throughput becomes the greatest after 57 rounds. The less rapid death of nodes in EEDBR makes its throughput greater than DBR after 100 rounds when most of the lowest depth nodes die in DBR.

The comparison of total number of packet drop is shown in Fig. 9. By virtue of the slowest rate of nodes death and adapting the path of the least interference in packet forwarding, EEIAR has the lowest packet drop as compared to the competitor schemes. For the first 207 rounds, DBR has greater packet drop than EEDBR due to faster death of the forwarder nodes. Beyond this, all nodes are dead in DBR that makes its packet drop constant while the corresponding packet drop in EEDBR increases as several nodes are still alive. The Fig. 10 compares the packet delivery ratio. This parameter is the highest for the proposed scheme due to the lowest packet drop and partially the highest number of packets received at the sink as described above. Finally, Fig. 11 shows the end-to-end delay. For the first 100 rounds, the proposed scheme has the lowest end-to-end delay due to the involvement of the least number of forwarder nodes. After that, its delay becomes greater than DBR because more nodes are alive in our proposed scheme that takes part in data routing as compared to DBR in which most of the nodes are dead. After 200 rounds, delay becomes greater in EEIAR than EEDBR because of greater number of alive nodes in the former that route the packets unless they all die at almost 500 rounds. For the first 50 rounds, DBR and EEDBR have the same end-to-end delay due to the availability of more forwarder nodes in both schemes. As number of rounds increases, nodes die faster in DBR than EEDBR so less number of nodes remains alive in the former to forward packets. As a result, delay becomes greater in the latter due to more alive nodes available for data forwarding.

\section{Conclusion}

We propose the EEIAR protocol for UWSNs. The parameters of depth and number of neighbors are used to select the forwarder nodes in routing data packets from source to destination. At every stage of routing, the decision of selecting a forwarder node is accomplished by the sender node rather than the receiver. A sender node selects a forwarder node among its neighbors with the lowest depth and the least number of neighbors. The lowest depth ensures that a packet comes closer to destination after each transmission. The least number of neighbors avoids interference, packet collision and packet drop at the network layer. The protocol reveals better performance in terms of the mentioned parameters as compared to some of the prevaling schemes. As a future work, more sinks can be used with path aware mobility to reduce the rapid death of sensor nodes and make the network lifetime longer.

\section{Acknowledgement}

This research work was partially supported by the Faculty of Computer Science and Information Technology, University of Malaya, under a special allocation of Postgraduate Fund. 


\section{References}

[1] M. H. Anisi, J. Rezazadeh and M. Dehghan, "FEDA: fault-tolerant energy-efficient data aggregation in wireless sensor networks," in Proc. of IEEE 16th Int. Con. on Software, Telecommunication and Computer Networks, pp.188-192, September 25-27, 2008.

Article (CrossRef Link).

[2] S. Kalhor, M. Anisi and A. T. Haghighat, "A new position-based routing protocol for reducing the number of exchanged route request messages in Mobile Ad-hoc networks,” in Proc. of IEEE 2nd Int. Con. on Systems and Networks Communications, pp.13-13, August 25-31, 2007. Article (CrossRef Link).

[3] Y. Zhou, Q. Li and G. Huo, "Human visual system based automatic underwater image enhancement in NSCT domain,” KSII Transactions on Internet and Information Systems, vol. 10, no. 2, pp. 837-856, February, 2016. Article (CrossRef Link).

[4] A. Khasawneh, M. Ahmad, A. Latif, M. shafie, C. Hasan T. Moeenuddin and B. Abdullah, "Pressure based routing protocol for underwater wireless sensor networks: a survey," KSII Transactions on Internet and Information Systems, vol. 9, no. 2, pp. 504-527, February, 2015. Article (CrossRef Link).

[5] J. Partan, J. Kurose and B. N. Levine, "Three-dimensional routing in underwater acoustic sensor networks," in Proc. of ACM 1st Int. Workshop on UnderWater Networks, pp. 17-24, September 25-25, 2006. Article (CrossRef Link).

[6] D. Pompili and M. Tommaso, "Three-dimensional routing in underwater acoustic sensor networks," in Proc. of ACM 2nd Int. Workshop on Performance Evaluation of Wireless Ad Hoc, Sensor, and Ubiquitous Networks, pp. 214-221, October 10-13, 2005. Article (CrossRef Link).

[7] J. Heidemann, W. Ye, J. Wills, A. Syed and Y. Li, "Research challenges and applications for underwater sensor networking," in Proc. of IEEE Wireless Communications and Networking Conference, pp. 1-8, April 3-6, 2006. Article (CrossRef Link).

[8] I. F. Akyildiz, D. Pompili and T. Melodia, "Underwater acoustic sensor networks: research challenges,” Ad Hoc Networks, vol. 3, no. 3, pp. 257-279, March, 2005. Article (CrossRef Link).

[9] H. Yan, Z. Shi and J. Cui, "DBR: depth-based routing for underwater sensor networks," in Proc. of IFIP-TC6 7th Int. Con. on Ad Hoc and Sensor Networks, Wireless Networks, Next Generation Internet, pp. 72-86, May 5-9, 2008. Article (CrossRef Link).

[10] D. Hwang and D. Kim, "DFR: directional flooding-based routing protocol for underwater sensor networks,” in Proc. of IEEE OCEANS, pp. 214-221, September 15-18, 2005. Article (CrossRef Link).

[11] P. Xie, J. Cui, and L. Lao, "VBF: vector-based forwarding protocol for underwater sensor networks," in Proc. of IFIP-TC6 5th Int. Con. on Networking Technologies, Services, and Protocols; Performance of Computer and Communication Networks; Mobile and Wireless Communications Systems, pp. 1216-1221, May 15-19, 2006. Article (CrossRef Link).

[12] N. Nicolaou, A. See, P. Xie, J. Cui and D. Maggiorini, "Improving the robustness of location-based routing for underwater sensor networks,” in Proc. of IEEE OCEANS, pp. 1-6, June 18-21, 2007. Article (CrossRef Link).

[13] J. Jornet, M. Stojanovic and M. Zorzi, "Focused beam routing protocol for underwater acoustic networks," in Proc. of ACM 3rd Int. Workshop on Underwater Networks, pp. 75-82, September 15-15, 2008. Article (CrossRef Link).

[14] A. Wahid, S. Lee, H. Jeong and D. Kim, "Eedbr: energy-efficient depth-based routing protocol for underwater wireless sensor networks," Advanced Computer Science and Information Technology, pp. 223-234, September, 2012. Article (CrossRef Link).

[15] X. Yang, Underwater Acoustic Sensor Networks, CRC Press, Florida, 2010. Article (CrossRef Link).

[16] A. Shashaj, R. Petroccia and C. Petrioli, "Energy efficient interference-aware routing and scheduling in underwater sensor networks," in Proc. of IEEE OCEANS, pp. 1-8, September 14-19, 2014. Article (CrossRef Link).

[17] A. Majid, I. Azam, T. Khan, Sangeen, Z. A. Khan, U. Qasim and N. Javaid, “A reliable and 
interference-aware routing protocol for underwater wireless sensor networks," in Proc. of IEEE 10th Int. Con. on Complex, Intelligent and Software Intensive Systems, pp. 247-255, July 6-8, 2016. Article (CrossRef Link).

[18] M. Shah, N. Javaid, M. Imran, M. Guizani, Z. A. Khan and U. Qasim, "Interference aware inverse EEDBR protocol for underwater WSNs," in Proc. of IEEE 11th Int. Con. on Wireless Communications and Mobile Computing Broadband, pp. 739-744, August 24-28, 2015. Article (CrossRef Link).

[19] U. Shakeel, N. Javaid, M. Ejaz, S. Zarar, T. Hafeez and Muhammad, "Improved interference-aware EEDBR protocol for underwater wireless sensor networks," in Proc. of IEEE 10th Int. Con. on Broadband and Wireless Computing, Communication and Applications, pp. 232-239, November 4-6 2015. Article (CrossRefL ink).

[20] H. Maqsood, N. Javaid, M. Z. Abidin, M. Ejaz, Muhammad and U. Shakeel, "Energy balanced interference aware energy efficient depth based routing protocol for UWSNs," in Proc. of IEEE 10th Int. Con. on Broadband and Wireless Computing, Communication and Applications, pp. 13-19, November 4-6, 2015. Article (CrossRef Link).

[21] A. Khan, N. Javaid, H. Mahmood, S. Khan, Z-A. Khan and U. Qasim, "An energy efficient interference and route aware protocol for WSNs," in Proc. of IEEE 10th Int. Con. on Complex, Intelligent and Software Intensive Systems, pp. 247-255, July 6-8, 2016. Article (CrossRef Link).

[22] Y-D. Chen, Y-W. Chen, C-Y. Lien and K-P. Shin, "A channel-aware depth-adaptive routing protocol for underwater acoustic sensor networks," in Proc. of IEEE OCEANS, pp. 1-6, April 7-10, 2014. Article (CrossRef Link).

[23] H. Fahim, N. Javaid, U. Qasim, Z. A. Khan, S. Javaid, A. Hayat, Z. Iqbal and G. Rehman, "Interference and bandwidth aware depth based routing protocols in underwater WSNs," in Proc. of IEEE 9th Int. Con. on Innovative Mobile and Internet Services in Ubiquitous Computing, pp. 78-85, July 8-10, 2015. Article (CrossRef Link).

[24] R. Urick, Principles of Underwater Sound for Engineers, McGraw Hill, New York, 1967.

[25] P. Etter, Underwater Aacoustic Modeling and Simulation, CRC Press, Florida, 2013. Article (CrossRef Link).

[26] K. V. Mackenzie, "Nine-term equation for sound speed in the oceans," Journal of Acoustic Society of America, vol. 9, no. 3, pp. 807-812, September, 1981. Article (CrossRef Link).

[27] A. Caruso, F. Paparella, L. F. M. Vieira, M. Erol and M. Gerla, "The meandering current mobility model and its impact on underwater mobile sensor networks," in Proc. of IEEE Int. Con. on Computer Communications, pp. 771-779, April 13-18, 2008. Article (CrossRef Link).

[28] D. Shin and D. Kim, "A dynamic NAV determination protocol in 802.11 based underwater networks," in Proc. of IEEE 5th Int. Symposium on Wireless Communication Systems, pp. 401-405, October 21-24, 2008. Article (CrossRef Link). 

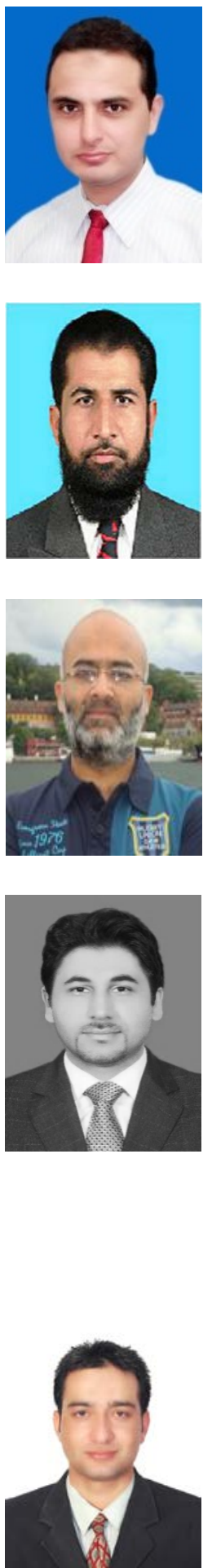

Mr. Anwar Khan received the M.Sc electronics degree from the Department of Electronics, University of Peshawar and the M.Phil Electronics degree from Quaid-i-Azam University, Islamabad, Pakistan, in 2006 and 2009, respectively. He joined the Department of Electronics, University of Peshawar, in 2009 as a faculty member. Currently, he is pursuing his $\mathrm{PhD}$ electronics from the Department of Electronics, Quaid-i-Azam University, Islamabad, Pakistan. His current research interests include, underwater wireless sensor networks, relayed communications, energy harvesting and game-theoretic optimization.

Mr. Ihsan Ali is a PhD Student at the Faculty of Computer Science and Information Technology, University of Malaya. He received his MSc degree in 2005 from Hazara University Manshera, Pakistan and MS degree in 2008 in Computer System Engineering from GIK Institute. He has more than five year teaching and research experience in different countries, including Saudi Arabia, USA, Pakistan and Malaysia. Mr. Ihsan has served as a Technical Program Committee Member for the IWCMC 2017, AINIS 2017, Future 5V 2017and also organizer of the special session on fog computing in Future 5V 2017. His research interests include Wireless Sensor networks, Underwater Sensor Networks, Sensor Cloud and Fog Computing.

Dr. Nadeem Javaid completed his Ph.D. from the University of Paris-Est, France in 2010 with the thesis entitled, "Analysis and Design of Routing Link Metrics for Quality Routing in Wireless Multi-hop Networks”. Previously, he has completed Masters in Electronics from Quid-I-Azam University, Islamabad in 1999. Recently, he is working as Associate Professor, and founding head of the ComSens (Communications over Sensors) Research Group at the Department of Computer Science, COMSATS Institute of Information Technology, Islamabad, Pakistan. His research interests include, Ad-hoc, Vehicular, Body Area, Under Water Sensor Networks, Renewable \& Sustainable Energy, Energy Management and SDNs.

Mohammad Hossein Anisi is with the School of Computer Science and Electronic Engineering, University of Essex, United Kingdom. He received his Ph.D from Universiti Teknologi Malaysia (UTM) while being awarded as the best postgraduate student. He has worked as senior lecturer at the Faculty of Computer Science and Information Technology, University of Malaya. His research interests lie in the area of Internet of Things, wireless sensor networks and their applications, mobile Ad hoc networks, and intelligent transportation systems. He has also collaborated actively with researchers in several other disciplines of computer science. Dr. Anisi has published several papers in high quality journals and conferences. He is associate editor of the Ad Hoc \& Sensor Wireless Networks (SCIE) and the KSII Transactions on Internet and Information Systems (SCIE) journals. He is also active member of IEEE, ACM, International Association of Engineers (IAENG) and Institute of Research Engineers and Doctors (the IRED).

Dr. Atiq-Ur-Rahman was born in Dir Lower, Pakistan in 1981. He received the BS degree in computer science from University of Peshawar and MS degree in computer system engineering from GIK Institute of Engineering Sciences and Technology, Pakistan in 2004 and 2008, respectively. He completed his PhD in Information Technology from Universiti Teknologi PETRONAS, Malaysia in 2013. He worked as a system engineer in National database and registration authority Pakistan from 2004 to 2006. Currently, he is working an Assistant Professor and Head of computer science department in Northern Border University, Kingdom of Saudi Arabia. His current research interest focuses on the energy-efficient design, routing, deployment, coverage and Quality of service in wireless sensor networks. 


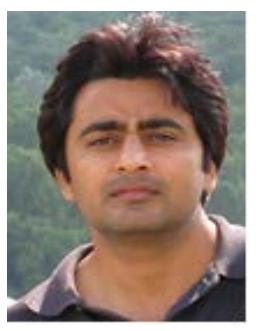

Naeem Bhatti received his MSc and MPhil degrees in electronics from Quaid-i-Azam University, Islamabad, Pakistan, in 2002 and 2004, respectively. He received his $\mathrm{PhD}$ degree in Informatics from the Vienna University of Technology, Vienna, Austria in 2012. He is currently with the Department of Electronics, Quaid-i-Azam University, as an assistant professor.

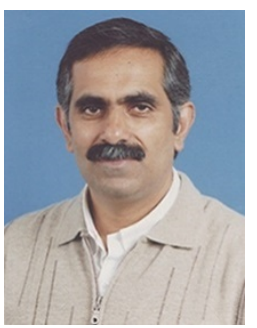

Muhammad Zia received his MS degree in electronics in 1991 and his MPhil in 1999, both from the Department of Electronics, Quaid-i-Azam University, Islamabad, Pakistan. He received his PhD degree in electrical engineering from the Department of Electrical and Computer Engineering, University of California, Davis, CA, USA, in 2010. He is currently with the Department of Electronics, Quaid-i-Azam University, as an assistant professor

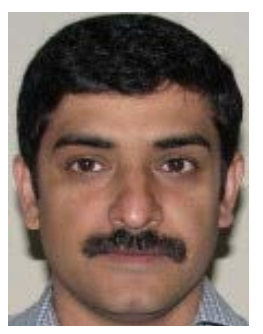

Hasan Mahmood received his MS degree in electronics from Quaid-i-Azam University, Islamabad, Pakistan, in 1991 and his MS degree in electrical engineering from the University of Ulm, Germany, in 2002. From 1994 to 2000, he was with the Department of Electronics, Quaid-i-Azam University, as a faculty member. He received his $\mathrm{PhD}$ degree in electrical engineering from the Stevens Institute of Technology, Hoboken, NJ, USA, in 2007. He is currently with the Department of Electronics, Quaid-i-Azam University, as an associate professor. 